\title{
Paraganglioma of the organ of Zuckerkandl and $F H$ gene mutation
}

\author{
Thiti Snabboon ${ }^{11,2}$, Natnicha Houngngam ${ }^{1}$, Sirinrat Tangjittrong ${ }^{2,3}$, Kewalee Sasiwimonphan ${ }^{4}$, \\ Kroonpong lampenkhae ${ }^{5}$, Supparerk Prichayudh ${ }^{6}$
}

${ }^{1}$ Excellence Centre in Diabetes, Hormone, and Metabolism, King Chulalongkorn Memorial Hospital, Thai Red Cross Society, Bangkok, Thailand

${ }^{2}$ Department of Medicine, Faculty of Medicine, Chulalongkorn University, Bangkok, Thailand

${ }^{3}$ Department of Medicine, Rayong Hospital, Rayong Province, Thailand

${ }^{4}$ Department of Radiology, Faculty of Medicine, Chulalongkorn University, Bangkok, Thailand

${ }^{5}$ Department of Pathology, Faculty of Medicine, Chulalongkorn University, Bangkok, Thailand

${ }^{6}$ Department of Surgery, Faculty of Medicine, Chulalongkorn University, Bangkok, Thailand

Key words: organs of ZuckerKandl; paraganglioma; FH gene

Paraganglioma (PGL) is an extra-adrenal chromaffin cell tumour. A sympathetic or functioning PGL (sPGL) is usually located in the thoracic, abdominal, and pelvic regions whereas a parasympathetic or non-functioning PGL is usually seen in the head and neck area. The organ of Zuckerkandl (OZ) comprises para-aortic paraganglia located between the level of inferior mesenteric artery and the aortic bifurcation, and this area is the most common site of abdominal PGL [1]. Strong genetic association with succinate dehydrogenase (SDHx) subunits, particularly $S D H B$ and $S D H D$, has previously been described [2].

The FH gene, a tumour suppressor gene located on chromosome 1q43, encodes fumarate hydratase, an immediate downstream of the SDH enzyme in the Krebs cycle. Its inactivating mutations predispose to hereditary leiomyomatosis and renal cell carcinoma (HLRCC; OMIM \# 150800) or Reed's syndrome and have recently been identified in pheochromocytoma/paraganglioma (PCC/PGL). Herein, we report a case of functioning OZ-PGL without the HLRCC features that carry the FH gene mutation.

A 33-year-old woman presented in 2009 with severe hypertension with orthostatic hypotension and a suprapubic mass. A functioning OZ-PGL was diagnosed by an elevated 24-hour urine metanephrine $(361.39 \mu \mathrm{g}$; normal 52-341 $\mu \mathrm{g}$ ) and 24-hour urine normetanephrine (810.29 $\mu \mathrm{g}$; normal 88-444 $\mu \mathrm{g}$ ). The patient subsequently underwent an uneventful tumour removal [3]. A ge- netic study was negative for the mutation in the classic PCC/PGL susceptibility genes (VHL, RET, SDHB, SDHC, and $S D H D)$. There was no evidence of relapse during follow-up visits. Ten years later, hypertension recurred and corresponded with her abnormal hormonal results: plasma metanephrine $93.41 \mathrm{pg} / \mathrm{mL}(0-96.64)$ and normetanephrine $509.35 \mathrm{pg} / \mathrm{mL}(0-163.05)$. A computerised tomography (CT) revealed a new lesion of the OZ-PGL, located slightly more superior than the previous one (Fig. 1). Additionally, 68Ga-DOTATATE positron emission tomography/computed tomography (PET/CT) scan did not show evidence of metastasis. Histopathological findings confirmed the diagnosis of PGL with a GAPP score of 3 (Fig. 2). The next-generation sequencing (NGS) and Sanger confirmatory showed a germline $\mathrm{FH}$ mutation, $\mathrm{c.817 \textrm {G }}>\mathrm{A}$ on exon 6; resulting in an alanine to threonine amino acid substitution in the conserved region of FH protein at the position 273, p.Ala273Thr. This de novo mutation was predicted "likely pathogenic" in accordance with the ACMG/AMP 2015 guideline because it is not present in either our in-house database of 4332 unrelated Thai exome alleles or her unaffected parents. The patient had no evidence of relapse during two-year follow-up periods, also without any clinical or radiological feature suggestive of HLRCC, cutaneous/uterine leiomyoma, and kidney cancer.

PCC/PGL is currently described as a tumour with the highest hereditability with at least one-third of the patients having a germline mutation. Moreover, several novel genes have been discovered with the 


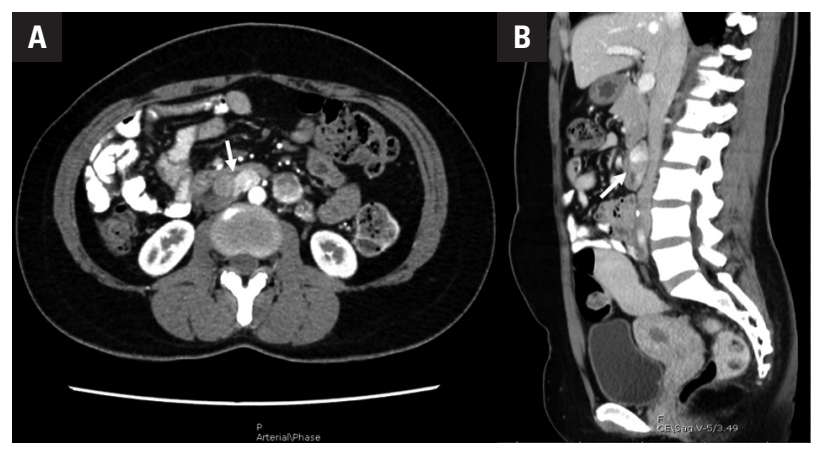

Figure 1. CT scan of the whole abdomen (A) arterial phase, axial view and (B) venous phase, sagittal view, shows multiple welldefined heterogeneous enhancing masses along bilateral para-aortic regions, causing anterior displacement of the third part of the duodenum (white arrow)

implementation of NGS technique. With recommendations for genetic testing in all PCC/PGL patients, particular phenotypes including syndromic presentations, extra-adrenal site or PGL, bilateral or multiple lesions, younger age, and malignancy are proposed as a high prevalence of genetic determinism [4]. PGL is strongly associated with pseudohypoxia-driven genes including SDH- and VHL/EPAS1-related genes. In this report we demonstrated a case of OZ-PGL carrying FH gene mutation, a member of $S D H$-related genes.

At least 150 cases of OZ-PGL have been reported, of which $70 \%$ present as functioning with sympathetic overactivity, particularly with norepinephrine predominance as described in our patient. A non-functioning tumour can be presented with abdominal pain or a palpable mass. While about $40 \%$ of sPGL carries a germline mutation, up to $70 \%$ of OZ-PGL has been associated with $S D H B$ and $S D H D[1,2]$. Because of its robust association with $S D H B$, its prevalence of malignancy is estimated to be at least $40 \%$, and about $10 \%$ of the patients already had metastasis at the time of diagnosis [1].

Clinical features of the $\mathrm{FH}$-associated PCC/PGL are not largely described because it accounts for only $1 \%$ of the genetically proven PCC/PGL [4]. Age at diagnosis ranges from 6 to 70 years [5]. About $40 \%$ of the patients have metastatic disease. Most mutations are missense and scattered throughout the gene. Given the common oncometabolic pathway of $S D H$ and $F H$, phenotypic overlap of both gene-related disorders in PCC/PGL is noted. It remains unclear whether individuals with $\mathrm{FH}$-related PCC/PGL are at high risk of developing other features of HLRCC; however, the FH mutations in PCC/PGL and HLRCC are in different regions of the gene.

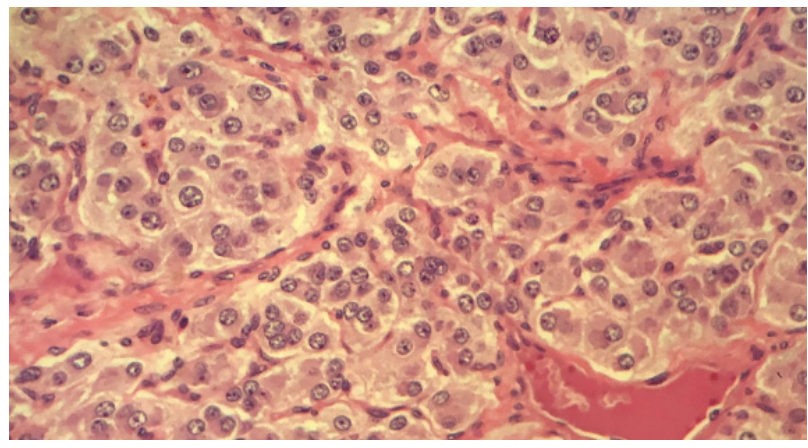

Figure 2. The histology of OZ is shown. Nest of neoplastic cell is surrounded by sustentacular cells (black arrow). The tumour cells possess mildly pleomorphic round to ovoid nuclei, granular chromatin, and basophilic cytoplasm; GAPP score of 3 . (Haematoxylin-eosin; ×100)

Our study confirms an association of the $\mathrm{FH}$ gene and PCC/PGL, particularly in the sPGL group, and recommends that the $F H$ gene be included in the genetic testing panel.

\section{Informed consent}

Informed consent was obtained from the patient for the publication of this article.

\section{Conflict of interest}

None declared.

\section{Acknowledgment}

The authors wish to thank Prof. Dr. Kanya Suphapeetiporn and Dr. Chupong Ittiwut for providing in-house Thai exome allele dataset, and Assist. Prof. Dr. Paisith Piriyawat and Assist. Prof. Dr. Patchaya Boonchaya-anant for editing the manuscript.

\section{References}

1. Lodish MB, Adams KT, Huynh TT, et al. Succinate dehydrogenase gene mutations are strongly associated with paraganglioma of the organ of Zuckerkandl. Endocr Relat Cancer. 2010; 17(3): 581-588, doi: 10.1677/ERC-10-0004, indexed in Pubmed: 20418362.

2. Castro-Vega LJ, Buffet A, De Cubas AA, et al. Germline mutations in FH confer predisposition to malignant pheochromocytomas and paragangliomas. Hum Mol Genet. 2014; 23(9): 2440-2446, doi: 10.1093/hmg/ddt639, indexed in Pubmed: 24334767.

3. Prichayudh S, Kritayakirana K, Snabboon T, et al. An extra-adrenal pheochromocytoma of the organ of Zuckerkandl: report of a case. J Med Assoc Thai. 2009; 92(11): 1532-1537, indexed in Pubmed: 19938747.

4. Lenders JWM, Duh QY, Eisenhofer G, et al. Endocrine Society. Pheochromocytoma and paraganglioma: an endocrine society clinical practice guideline. J Clin Endocrinol Metab. 2014; 99(6): 1915-1942, doi: 10.1210/jc.2014-1498, indexed in Pubmed: 24893135.

5. Clark GR, Sciacovelli M, Gaude E, et al. Germline FH mutations presenting with pheochromocytoma. J Clin Endocrinol Metab. 2014; 99(10): E2046-E2050, doi: 10.1210/jc.2014-1659, indexed in Pubmed: 25004247. 